\title{
EQUATIONS RELATED TO DERIVATIONS ON PRIME RINGS
}

\author{
MAJA Fošner AND Joso VuKMAN \\ University of Maribor, Slovenia \\ Dedicated to the memory of Professor Svetozar Kurepa
}

\begin{abstract}
In this paper we prove the following result. Let $m \geq 0$ and $n \geq 0$ be integers with $m+n \neq 0$ and let $R$ be a prime ring with $\operatorname{char}(R)=0$ or $m+n+1 \leq \operatorname{char}(R) \neq 2$. Suppose there exists a nonzero additive mapping $D: R \rightarrow R$ satisfying the relation $D\left(x^{m+n+1}\right)=(m+$ $n+1) x^{m} D(x) x^{n}$ for all $x \in R$. In this case $D$ is a derivation and $R$ is commutative.
\end{abstract}

Throughout this paper $R$ will represent an associative ring with center $Z(R)$. Given an integer $n>1$, a ring $R$ is said to be $n$-torsion free if for $x \in R$, $n x=0$ implies $x=0$. As usual the commutator $x y-y x$ will be denoted by $[x, y]$. Recall that a ring $R$ is prime if $a R b=(0)$ implies that either $a=0$ or $b=0$, and is semiprime if $a R a=(0)$ implies $a=0$. An additive mapping $D: R \rightarrow R$ is called a derivation if $D(x y)=D(x) y+x D(y)$ holds for all pairs $x, y \in R$. A mapping $F$ of a ring $R$ into itself is called commuting on $R$ in case $[F(x), x]=0$ holds for all $x \in R$. A classical result of Posner ([10]) states that the existence of a nonzero commuting derivation $D: R \rightarrow R$, where $R$ is a prime ring, forces the ring to be commutative.

Let us start with the following result.

Proposition 1. Let $R$ be a noncommutative prime ring with char $(R) \neq 2$ and let $D: R \rightarrow R$ be a nonzero additive mapping satisfying the relation

$$
D\left(x^{2}\right)=2 x D(x),
$$

for all $x \in R$. In this case $D=0$.

2010 Mathematics Subject Classification. 16N60, 39B05.

Key words and phrases. Prime ring, functional identity, derivation. 
The result above has been first proved by Brešar and Vukman ([8, Corollary 1.3]) under the additional assumption that $\operatorname{char}(R) \neq 3$. Later on Deng ([9]) removed the assumption that $\operatorname{char}(R) \neq 3$.

We proceed with the result below proved by Vukman ([12]).

Proposition 2. Let $R$ be a noncommutative prime ring with the identity element and $\operatorname{char}(R) \neq 2, \operatorname{char}(R) \neq 3$, and let $D: R \rightarrow R$ be a nonzero additive mapping satisfying the relation

$$
D\left(x^{3}\right)=3 x D(x) x,
$$

for all $x \in R$. In this case $D=0$.

Recently, Vukman ([13]) has proved the following theorem.

Proposition 3. Let $R$ be a 2-torsion free semiprime ring and let $D$ : $R \rightarrow R$ be an additive mapping satisfying the relation (1) for all $x \in R$. In this case $D$ is a derivation which maps $R$ into $Z(R)$.

The observations above lead to the following conjecture:

Let $m \geq 0$ and $n \geq 0$ be integers with $m+n \neq 0$ and let $R$ be a semiprime ring with suitable torsion restrictions. Suppose there exists an additive mapping $D: R \rightarrow R$ satisfying the relation

$$
D\left(x^{m+n+1}\right)=(m+n+1) x^{m} D(x) x^{n}
$$

for all $x \in R$. In this case $D$ is a derivation which maps $R$ into $Z(R)$.

Vukman and Kosi-Ulbl ([15]) have recently proved the above conjecture in case $R$ is a semiprime ring with the identity element. It is our aim in this paper to prove the following result which is related to the conjecture above.

TheOREM 4. Let $m \geq 0$ and $n \geq 0$ be integers with $m+n \neq 0$ and let $R$ be a prime ring with $\operatorname{char}(R)=0$ or $m+n+1 \leq \operatorname{char}(R) \neq 2$. Suppose there exists a nonzero additive mapping $D: R \rightarrow R$ satisfying the relation

$$
D\left(x^{m+n+1}\right)=(m+n+1) x^{m} D(x) x^{n}
$$

for all $x \in R$. In this case $D$ is a derivation and $R$ is commutative.

For the proof of Theorem 4 we need Theorem 5 below, which is of independent interest. Our result is obtained as an application of the theory of functional identities.

The theory of functional identities considers set-theoretic mappings on rings satisfying some identical relation. When treating such relations one usually concludes that the form of the mappings involved can be described, unless the ring is very special. We refer the reader to [7] for introductory account on functional identities, where Brešar presents this new theory, the theory of (generalized) functional identities, and its applications, to a wider audience. 
Let $R$ be a ring and let $X$ be a subset of $R$. By $C(X)$ we denote the set $\{r \in R \mid[r, X]=0\}$. Let $m \in \mathbb{N}$ and let $E: X^{m-1} \rightarrow R, p: X^{m-2} \rightarrow R$ be arbitrary mappings. In the case when $m=1$ this should be understood as that $E$ is an element in $R$ and $p=0$. Let $1 \leq i<j \leq m$ and define $E^{i}, p^{i j}, p^{j i}: X^{m} \rightarrow R$ by

$$
\begin{aligned}
E^{i}\left(\bar{x}_{m}\right) & =E\left(x_{1}, \ldots, x_{i-1}, x_{i+1}, \ldots, x_{m}\right), \\
p^{i j}\left(\bar{x}_{m}\right)=p^{j i}\left(\bar{x}_{m}\right) & =\left(x_{1}, \ldots, x_{i-1}, x_{i+1}, \ldots, x_{j-1}, x_{j+1}, \ldots, x_{m}\right),
\end{aligned}
$$

where $\bar{x}_{m}=\left(x_{1}, \ldots, x_{m}\right) \in X^{m}$.

Let $I, J \subseteq\{1, \ldots, m\}$, and for each $i \in I, j \in J$ let $E_{i}, F_{j}: X^{m-1} \rightarrow R$ be arbitrary mappings. Consider functional identities

$$
\begin{aligned}
& \sum_{i \in I} E_{i}^{i}\left(\bar{x}_{m}\right) x_{i}+\sum_{j \in J} x_{j} F_{j}^{j}\left(\bar{x}_{m}\right)=0 \quad\left(\bar{x}_{m} \in X^{m}\right), \\
& \sum_{i \in I} E_{i}^{i}\left(\bar{x}_{m}\right) x_{i}+\sum_{j \in J} x_{j} F_{j}^{j}\left(\bar{x}_{m}\right) \in C(X) \quad\left(\bar{x}_{m} \in X^{m}\right) .
\end{aligned}
$$

A natural possibility when (4) and (5) are fulfilled is when there exist mappings $p_{i j}: X^{m-2} \rightarrow R, i \in I, j \in J, i \neq j$, and $\lambda_{k}: X^{m-1} \rightarrow C(X)$, $k \in I \cup J$, such that

$$
\begin{aligned}
& E_{i}^{i}\left(\bar{x}_{m}\right)=\sum_{j \in J, j \neq i} x_{j} p_{i j}^{i j}\left(\bar{x}_{m}\right)+\lambda_{i}^{i}\left(\bar{x}_{m}\right), \\
& F_{j}^{j}\left(\bar{x}_{m}\right)=-\sum_{i \in I, j \neq i} p_{i j}^{i j}\left(\bar{x}_{m}\right) x_{i}-\lambda_{j}^{j}\left(\bar{x}_{m}\right), \\
& \lambda_{k}=0 \quad \text { if } \quad k \notin I \cap J
\end{aligned}
$$

for all $\bar{x}_{m} \in X^{m}, i \in I, j \in J$. We shall say that every solution of the form (6) is a standard solution of (4) and (5).

The case when one of the sets $I$ or $J$ is empty is not excluded. The sum over the empty set of indexes should be simply read as zero. So, when $J=0$ (resp. $I=0)(4)$ and $(5)$ reduce to

$$
\begin{aligned}
& \text { (7) } \sum_{i \in I} E_{i}^{i}\left(\bar{x}_{m}\right) x_{i}=0 \quad\left(\text { resp. } \sum_{j \in J} x_{j} F_{j}^{j}\left(\bar{x}_{m}\right)=0\right) \quad\left(\bar{x}_{m} \in X^{m}\right), \\
& \text { (8) } \sum_{i \in I} E_{i}^{i}\left(\bar{x}_{m}\right) x_{i} \in C(X) \quad\left(\text { resp. } \sum_{j \in J} x_{j} F_{j}^{j}\left(\bar{x}_{m}\right) \in C(X)\right) \quad\left(\bar{x}_{m} \in X^{m}\right) .
\end{aligned}
$$

In that case the (only) standard solution is

$$
E_{i}=0, \quad i \in I \quad\left(\text { resp. } F_{j}=0, \quad j \in J\right) .
$$

A $d$-freeness of $X$ will play an important role in this paper. For a definition of $d$-freeness we refer the reader to [5]. Under some natural assumptions one can establish that various subsets (such as ideals, Lie ideals, the sets of symmetric or skew symmetric elements in a ring with involution) of certain 
types of rings are $d$-free. We refer the reader to [1] and [2] for results of this kind. Let us mention that a prime ring $R$ is a $d$-free subset of its maximal right ring of quotients, unless $R$ satisfies the standard polynomial identity of degree less than $2 d$ (see [2, Theorem 2.4]).

Let $R$ be a ring and let

$$
p\left(x_{1}, x_{2}, \ldots, x_{m+n+1}\right)=\sum_{\pi \in S_{m+n+1}} x_{\pi(1)} x_{\pi(2)} \cdot \ldots \cdot x_{\pi(m+n+1)}
$$

be a fixed multilinear polynomial in noncommutative indeterminates $x_{1}, x_{2}$, $\ldots, x_{m+n+1}$. Further, let $L$ be a subset of $R$ closed under $p$, i.e., $p\left(\bar{x}_{m+n+1}\right) \in$ $L$ for all $x_{1}, x_{2}, \ldots, x_{m+n+1} \in L$, where $\bar{x}_{n}=\left(x_{1}, x_{2}, \ldots, x_{m+n+1}\right)$. We shall consider a mapping $D: L \rightarrow R$ satisfying

$$
\begin{aligned}
& D\left(p\left(\bar{x}_{m+n+1}\right)\right)= \\
& (m+n+1) \sum_{\pi \in S_{m+n+1}} x_{\pi(1)} \ldots x_{\pi(m)} D\left(x_{\pi(m+1)}\right) x_{\pi(m+2)} \ldots x_{\pi(m+n+1)}
\end{aligned}
$$

for all $x_{1}, x_{2}, \ldots, x_{m+n+1} \in L$. Of course, every two-sided centralizer satisfies (10). Our goal is to show that under certain assumptions these are in fact the only mappings with this property. In the first step of the proof we derive a functional identity from (10). Let us mention that the idea of considering the expression $\left[p\left(\bar{x}_{n}\right), p\left(\bar{y}_{n}\right)\right]$ in its proof is taken from [3].

TheOREM 5. Let $L$ be a $2(m+n+1)$-free Lie subring of $R$ closed under p. If $D: L \rightarrow R$ is an additive mapping satisfying (10), then there exist $p \in C(L)$ and $\lambda: L \rightarrow C(L)$ such that $D(x)=p x+\lambda(x)$ for all $x \in L$.

Proof. Let us write $k=m+n+1$ for brevity. Note that for any $a \in R$ and $\bar{x}_{k} \in L^{k}$ we have

$$
\left[p\left(\bar{x}_{k}\right), a\right]=\sum_{i=1}^{k} p\left(x_{1}, \ldots, x_{i-1},\left[x_{i}, a\right], x_{i+1}, \ldots, x_{k}\right) .
$$

Using (10) it follows that

$$
\begin{aligned}
& D\left[p\left(\bar{x}_{k}\right), a\right]= \\
& \quad k \sum_{\pi \in S_{k}}\left[x_{\pi(1)}, a\right] x_{\pi(2)} \ldots x_{\pi(m)} D\left(x_{\pi(m+1)}\right) x_{\pi(m+2)} \ldots x_{\pi(k)} \\
& \quad+k \sum_{\pi \in S_{k}} x_{\pi(1)}\left[x_{\pi(2)}, a\right] x_{\pi(3)} \ldots x_{\pi(m)} D\left(x_{\pi(m+1)}\right) x_{\pi(m+2)} \ldots x_{\pi(k)} \\
& \quad+\ldots+k \sum_{\pi \in S_{k}} x_{\pi(1)} \ldots x_{\pi(m)} D\left[x_{\pi(m+1)}, a\right] x_{\pi(m+2)} \ldots x_{\pi(k)} \\
& \quad+\ldots+k \sum_{\pi \in S_{k}} x_{\pi(1)} \ldots x_{\pi(m)} D\left(x_{\pi(m+1)}\right) x_{\pi(m+2)} \ldots\left[x_{\pi(k)}, a\right]
\end{aligned}
$$


Hence

$$
\begin{aligned}
& D\left[p\left(\bar{x}_{k}\right), a\right]= \\
& \quad k \sum_{\pi \in S_{k}}\left[x_{\pi(1)} \ldots x_{\pi(m)}, a\right] D\left(x_{\pi(m+1)}\right) x_{\pi(m+2)} \ldots x_{\pi(k)} \\
& \quad+k \sum_{\pi \in S_{k}} x_{\pi(1)} \ldots x_{\pi(m)} D\left[x_{\pi(m+1)}, a\right] x_{\pi(m+2)} \ldots x_{\pi(k)} \\
& \quad+k \sum_{\pi \in S_{k}} x_{\pi(1)} \ldots x_{\pi(m)} D\left(x_{\pi(m+1)}\right)\left[x_{\pi(m+2)} \ldots x_{\pi(k)}, a\right] .
\end{aligned}
$$

In particular

$$
\begin{aligned}
& D\left[p\left(\bar{x}_{k}\right), p\left(\bar{y}_{k}\right)\right]= \\
& \quad k \sum_{\pi \in S_{k}}\left[x_{\pi(1)} \ldots x_{\pi(m)}, p\left(\bar{y}_{k}\right)\right] D\left(x_{\pi(m+1)}\right) x_{\pi(m+2)} \ldots x_{\pi(k)} \\
& \quad+k \sum_{\pi \in S_{k}} x_{\pi(1)} \ldots x_{\pi(m)} D\left[x_{\pi(m+1)}, p\left(\bar{y}_{k}\right)\right] x_{\pi(m+2)} \ldots x_{\pi(k)} \\
& \quad+k \sum_{\pi \in S_{k}} x_{\pi(1)} \ldots x_{\pi(m)} D\left(x_{\pi(m+1)}\right)\left[x_{\pi(m+2)} \ldots x_{\pi(k)}, p\left(\bar{y}_{k}\right)\right]
\end{aligned}
$$

for all $\bar{x}_{k}, \bar{y}_{k} \in L^{k}$. We also have (by (11))

$$
\begin{aligned}
& \varphi\left(x_{\pi(m+1)}\right)=D\left[x_{\pi(m+1)}, p\left(\bar{y}_{k}\right)\right]=-D\left[p\left(\bar{y}_{k}\right), x_{\pi(m+1)}\right] \\
& \quad k \sum_{\sigma \in S_{k}}\left[x_{\pi(m+1)}, y_{\sigma(1)} \ldots y_{\sigma(m)}\right] D\left(y_{\sigma(m+1)}\right) y_{\sigma(m+2)} \ldots y_{\sigma(k)} \\
& \quad+k \sum_{\sigma \in S_{k}} y_{\sigma(1)} \ldots y_{\sigma(m)} D\left[x_{\pi(m+1)}, y_{\sigma(m+1)}\right] y_{\sigma(m+2)} \ldots y_{\sigma(k)} \\
& \quad+k \sum_{\sigma \in S_{k}} y_{\sigma(1)} \ldots y_{\sigma(m)} D\left(y_{\sigma(m+1)}\right)\left[x_{\pi(m+1)}, y_{\sigma(m+2)} \ldots y_{\sigma(k)}\right]
\end{aligned}
$$

for all $\bar{y}_{k} \in L^{k}$. Therefore (12) can be written as

$$
\begin{aligned}
& D\left[p\left(\bar{x}_{k}\right), p\left(\bar{y}_{k}\right)\right]= \\
& \quad k \sum_{\pi \in S_{k}}\left[x_{\pi(1)} \ldots x_{\pi(m)}, p\left(\bar{y}_{k}\right)\right] D\left(x_{\pi(m+1)}\right) x_{\pi(m+2)} \ldots x_{\pi(k)} \\
& \quad+k \sum_{\pi \in S_{k}} x_{\pi(1)} \ldots x_{\pi(m)} \varphi\left(x_{\pi(m+1)}\right) x_{\pi(m+2)} \ldots x_{\pi(k)} \\
& \quad+k \sum_{\pi \in S_{k}} x_{\pi(1)} \ldots x_{\pi(m)} D\left(x_{\pi(m+1)}\right)\left[x_{\pi(m+2)} \ldots x_{\pi(k)}, p\left(\bar{y}_{k}\right)\right]
\end{aligned}
$$


for all $\bar{x}_{k}, \bar{y}_{k} \in L^{k}$. On the other hand, using $\left[p\left(\bar{x}_{k}\right), p\left(\bar{y}_{k}\right)\right]=-\left[p\left(\bar{y}_{k}\right), p\left(\bar{x}_{k}\right)\right]$, we get from the above identity

$$
\begin{aligned}
& D\left[p\left(\bar{x}_{k}\right), p\left(\bar{y}_{k}\right)\right]= \\
& \quad k \sum_{\sigma \in S_{k}}\left[p\left(\bar{x}_{k}\right), y_{\sigma(1)} \ldots y_{\sigma(m)}\right] D\left(y_{\sigma(m+1)}\right) y_{\sigma(m+2)} \ldots y_{\sigma(k)} \\
& \quad+k \sum_{\sigma \in S_{k}} y_{\sigma(1)} \ldots y_{\sigma(m)} \varphi^{\prime}\left(y_{\sigma(m+1)}\right) y_{\sigma(m+2)} \ldots y_{\sigma(k)} \\
& \quad+k \sum_{\sigma \in S_{k}} y_{\sigma(1)} \ldots y_{\sigma(m)} D\left(y_{\sigma(m+1)}\right)\left[p\left(\bar{x}_{k}\right), y_{\sigma(m+2)} \ldots y_{\sigma(k)}\right]
\end{aligned}
$$

for all $\bar{x}_{k}, \bar{y}_{k} \in L^{k}$, where

$$
\begin{aligned}
& \varphi^{\prime}\left(y_{\sigma(m+1)}\right)= \\
& \quad k \sum_{\pi \in S_{k}}\left[x_{\pi(1)} \ldots x_{\pi(m)}, y_{\sigma(m+1)}\right] D\left(x_{\pi(m+1)}\right) x_{\pi(m+2)} \ldots x_{\pi(k)} \\
& \quad+k \sum_{\pi \in S_{k}} x_{\pi(1)} \ldots x_{\pi(m)} D\left[x_{\pi(m+1)}, y_{\sigma(m+1)}\right] x_{\pi(m+2)} \ldots x_{\pi(k)} \\
& \quad+k \sum_{\pi \in S_{k}} x_{\pi(1)} \ldots x_{\pi(m)} D\left(x_{\pi(m+1)}\right)\left[x_{\pi(m+2)} \ldots x_{\pi(k)}, y_{\sigma(m+1)}\right]
\end{aligned}
$$

Let $s: \mathbb{Z} \rightarrow \mathbb{Z}$ be a mapping defined by $s(i)=i-k$. For each $\sigma \in S_{k}$ the mapping $s^{-1} \sigma s:\{k+1, \ldots, 2 k\} \rightarrow\{k+1, \ldots, 2 k\}$ will be denoted by $\bar{\sigma}$. After comparing above identities and writing $x_{k+i}$ instead of $y_{i}, i=1, \ldots, k$, we arrive at

$$
\begin{aligned}
0= & \sum_{\pi \in S_{k}} \sum_{\sigma \in S_{k}} k\left(\left[x_{\pi(1)} \ldots x_{\pi(k)}, x_{\bar{\sigma}(k+1)} \ldots x_{\bar{\sigma}(k+m)}\right] D\left(x_{\bar{\sigma}(k+m+1)}\right)\right. \\
& +x_{\bar{\sigma}(k+1)} \ldots x_{\bar{\sigma}(k+m)} \varphi\left(x_{\bar{\sigma}(k+m+1)}\right) \\
& +x_{\bar{\sigma}(k+1)} \ldots x_{\bar{\sigma}(k+m)} D\left(x_{\bar{\sigma}(k+m+1)}\right) x_{\pi(1)} \ldots x_{\pi(k)} \\
& \left.-x_{\pi(1)} \ldots x_{\pi(m)} D\left(x_{\pi(m+1)}\right) x_{\pi(m+2)} \ldots x_{\pi(k)} x_{\bar{\sigma}(k+1)} \ldots x_{\bar{\sigma}(k+m+1)}\right) \\
& x_{\bar{\sigma}(k+m+2)} \ldots x_{\bar{\sigma}(2 k)} \\
- & \sum_{\pi \in S_{2 k}} \sum_{\sigma \in S_{k}} k\left(\left[x_{\pi(1)} \ldots x_{\pi(m)}, x_{\bar{\sigma}(k+1)} \ldots x_{\bar{\sigma}(2 k)}\right] D\left(x_{\pi(m+1)}\right)\right. \\
& +x_{\pi(1)} \ldots x_{\pi(m)} \varphi\left(x_{\pi(m+1)}\right) \\
& +x_{\bar{\sigma}(k+1)} \ldots x_{\bar{\sigma}(k+m)} D\left(x_{\bar{\sigma}(k+m+1)}\right) x_{\bar{\sigma}(k+m+2)} \ldots x_{\bar{\sigma}(2 k)} x_{\pi(1)} \ldots x_{\pi(m+1)} \\
& \left.-x_{\pi(1)} \ldots x_{\pi(m)} D\left(x_{\pi(m+1)}\right) x_{\bar{\sigma}(k+1)} \ldots x_{\bar{\sigma}(2 k)}\right) x_{\pi(m+2)} \ldots x_{\pi(k)} .
\end{aligned}
$$


Since $L$ is $2 k$-free, it follows that so obtained functional identity has only a standard solution. In particular,

$$
\begin{aligned}
0= & \sum_{\pi \in S_{k}} \sum_{\sigma \in S_{m+1}} k\left(\left[x_{\pi(1)} \ldots x_{\pi(k)}, x_{\bar{\sigma}(k+1)} \ldots x_{\bar{\sigma}(k+m)}\right] D\left(x_{\bar{\sigma}(k+m+1)}\right)\right. \\
& +x_{\bar{\sigma}(k+1)} \ldots x_{\bar{\sigma}(k+m)} \varphi\left(x_{\bar{\sigma}(k+m+1)}\right) \\
& +x_{\bar{\sigma}(k+1)} \ldots x_{\bar{\sigma}(k+m)} D\left(x_{\bar{\sigma}(k+m+1)}\right) x_{\pi(1)} \ldots x_{\pi(k)} \\
& \left.-x_{\pi(1)} \ldots x_{\pi(m)} D\left(x_{\pi(m+1)}\right) x_{\pi(m+2)} \ldots x_{\pi(k)} x_{\bar{\sigma}(k+1)} \ldots x_{\bar{\sigma}(k+m+1)}\right) .
\end{aligned}
$$

Note that this is also a functional identity. It follows that

$$
\begin{aligned}
& \sum_{\pi \in S_{k}} \sum_{\sigma \in S_{m+1}} k x_{\pi(m+1)} \ldots x_{\bar{\sigma}(k+m)} D\left(x_{\pi(k+m+1)}\right) \\
& -k D\left(x_{\pi(m+1)}\right) x_{\pi(m+2)} \ldots x_{\bar{\sigma}(k+m+1)}=0
\end{aligned}
$$

where $\pi(i)=i$ for all $i=1, \ldots, m$. After some steps we arrive at

$$
D(x)=x p+\lambda(x)
$$

for all $x \in L$, where $p \in R$ and $\lambda: R \rightarrow C(L)$. Similarly, by (13) we can prove

$$
D(x)=q x+\mu(x)
$$

for all $x \in L$, where $q \in R$ and $\mu: R \rightarrow C(L)$. Comparing (14) and (15) we arrive at

$$
x p-q x \in C(L)
$$

for all $x \in L$. It follows that $p=q \in C(L)$ and $\lambda=\mu$. Thereby the proof is completed.

We are now in the position to prove Theorem 4.

Proof. The complete linearization of (3) gives us (10).

First suppose that $R$ is not a PI ring (satisfying the standard polynomial identity of degree less than $4 k$ ). According to Theorem 5 there exist $p \in C$ and $\lambda: R \rightarrow C$ such that

$$
D(x)=p x+\lambda(x)
$$

for all $x \in R$. Using this with (10) we see that

$$
\sum_{\pi \in S_{k}} x_{\pi(1)} \ldots x_{\pi(k-1)}\left((k-1) p x_{\pi(k)}+k \lambda\left(x_{\pi(k)}\right)\right)=\lambda\left(p\left(\bar{x}_{k}\right)\right)
$$

for all $x_{1}, x_{2}, \ldots, x_{k} \in R$. Since $R$ is not a PI ring it follows that $\lambda(x)=0$

$$
(k-1) p x+k \lambda(x)=0
$$

for all $x \in R$. Thus $[(k-1) p x, y]=0$ for all $x, y \in R$, which in turn implies $[x, y] z p=0$ for all $x, y, z \in R$. By the primeness of $R$ it follows that $R$ is commutative or $p=0$. The second relation gives us $\lambda(x)=0$ for all $x \in R$ by $(16)$. Thus $D=0$. Suppose now that $[x, y]=0$ for all $x, y \in R$. Using 
(16) it follows that $\lambda(x) y-\lambda(y) x=0$ for all $x, y \in R$, which implies $\lambda=0$. Consequently $D=0$.

Assume now that $R$ is a PI ring. It is well-known that in this case $R$ has a nonzero center (see [11]). Let $c$ be a nonzero central element. Pick any $x \in R$ and set $x_{1}=x_{2}=\ldots=x_{k-1}=c x$ and $x_{k}=x$ in (10). We arrive at

$$
\begin{aligned}
D\left(k ! c^{k-1} x^{k}\right)= & k(n+m) ! c^{m+n-1}\left(x^{m} D(c x) x^{n} n+\right. \\
& \left.c x^{m} D(x) x^{n}+x^{m} D(c x) x^{n} m\right) .
\end{aligned}
$$

On the other hand, setting $x_{1}=x_{2}=\ldots=x_{k-1}=c$ and $x_{k}=x^{k}$ in (10) we obtain

$$
D\left(k ! c^{k-1} x^{k}\right)=k(n+m) ! c^{m+n-1}\left(c D\left(x^{k}\right)+x^{k} D(c) m+D(c) x^{k} n\right) .
$$

Comparing so obtained relations we get

$$
\begin{aligned}
& c(k-1) x^{m} D(x) x^{n}= \\
& n\left(x^{m} D(c x) x^{n}-D(c) x^{k}\right)+m\left(x^{m} D(c x) x^{n}-x^{k} D(c)\right)
\end{aligned}
$$

for all $x \in R$. In the case when $x=c$ we have

$$
D\left(c^{2}\right)=2 c D(c) .
$$

After the complete linearization of (17) and putting $x_{1}=x$ and $x_{2}=\ldots=$ $x_{k}=c$ in the so obtained identity we get

$$
(k-1)(D(c x)-c D(x))=D(c) x n+x D(c) m
$$

for all $x \in R$. Putting $x y, y \in R$, in this relation instead of $x$ we obtain

$$
(k-1)(D(c x y)-c D(x y))=D(c) x y n+x y D(c) m .
$$

Multiplying (19) by $y \in R$ on the right side we infer

$$
(k-1)(D(c x) y-c D(x) y)=D(c) x y n+x D(c) y m .
$$

Comparing so obtained identities, (20) and (21), we arrive at

$(22)(k-1)(D(c x y)-c D(x y)-D(c x) y+c D(x) y)=m x[y, D(c)]$

for all $x, y \in R$. Putting $y=c$ in this identity we get

$$
D\left(c^{2} x\right)=2 c D(c x)-c^{2} D(x)
$$

for all $x \in R$.

Putting $c x$ instead of $x$ in (3) we get

$$
D\left(c^{k} x^{k}\right)=k c^{k-1} x^{m} D(c x) x^{n}
$$

for all $x \in R$. On the other hand, setting $x_{1}=x_{2}=\ldots=x_{k-1}=c$ and $x_{k}=c x^{k}$ in (10) we obtain

$$
D\left(k ! c^{k} x^{k}\right)=k(n+m) ! c^{k-1}\left(D\left(c x^{k}\right)+x^{k} D(c) m+D(c) x^{k} n\right),
$$

which yields

$$
D\left(c^{k} x^{k}\right)=c^{k-1}\left(D\left(c x^{k}\right)+x^{k} D(c) m+D(c) x^{k} n\right)
$$


for all $x \in R$. Comparing identities (24) and (25) we arrive at

$$
k x^{m} D(c x) x^{n}=D\left(c x^{k}\right)+x^{k} D(c) m+D(c) x^{k} n
$$

for all $x \in R$. Using (19) it follows that

$$
\begin{aligned}
(k-1) k x^{m} D(c x) x^{n}= & (k-1) D\left(c x^{k}\right)+(k-1) x^{k} D(c) m+(k-1) D(c) x^{k} n \\
= & (k-1) c D\left(x^{k}\right)+n D(c) x^{k}+m x^{k} D(c) \\
& +(k-1) x^{k} D(c) m+(k-1) D(c) x^{k} n \\
= & (k-1) k c x^{m} D(x) x^{n}+n k D(c) x^{k}+m k x^{k} D(c),
\end{aligned}
$$

which in turn implies

$$
(k-1) x^{m}(D(c x)-c D(x)) x^{n}=n D(c) x^{k}+m x^{k} D(c)
$$

for all $x \in R$. Again using (19) we arrive at

$$
x^{m}(D(c) x n+x D(c) m) x^{n}=n D(c) x^{k}+m x^{k} D(c)
$$

for all $x \in R$. Thus we have

$$
n\left[x^{m}, D(c)\right] x^{n+1}=m x^{m+1}\left[x^{n}, D(c)\right]
$$

for all $x \in R$. After a complete linearization and putting $x_{1}=x_{2}=x$ and $x_{3}=\ldots=x_{k}=c$ in this indentity we obtain

$$
[[x, D(c)], x]=0
$$

for all $x \in R$. Using Posner's second theorem it follows that $[x, D(c)]=0$ for all $x \in R$. Putting $x=c$ in (22) and using (18) and (23) we get

$$
D(c x)=D(c) x+c D(x)
$$

for all $x \in R$.

Pick any $x \in R$ and set $x_{1}=x_{2}=\ldots=x_{k-2}=c$ and $x_{k-1}=x_{k}=x$ in (10). We arrive at

$$
\begin{aligned}
(k-1) D\left(c^{k-2} x^{2}\right) & =m(m-1) x^{2} c^{k-3} D(c)+2 m n c^{k-3} x D(c) x \\
& +2 n c^{k-2} D(x) x+n(n-1) c^{k-3} D(c) x^{2}+2 m c^{k-2} x D(x)
\end{aligned}
$$

for all $x \in R$. It is not difficult to prove that $D\left(c^{n}\right)=n c^{n-1} D(c)$ for all $n \in \mathbb{N}$. Consequently by $(27)$ we have

$$
(k-1) D\left(c^{k-2} x^{2}\right)=(k-1)\left((k-2) c^{k-3} D(c) x^{2}+c^{k-2} D\left(x^{2}\right)\right)
$$

for all $x \in R$. Comparing so obtained identities we arrive at

$$
\begin{aligned}
& (k-1)\left((k-2) D(c) x^{2}+c D\left(x^{2}\right)\right)=m(m-1) x^{2} D(c)+2 m n x D(c) x \\
& +2 n c D(x) x+n(n-1) D(c) x^{2}+2 m c x D(x),
\end{aligned}
$$

which yields

$$
(k-1) D\left(x^{2}\right)=2 n D(x) x+2 m x D(x)
$$

for all $x \in R$. 
Putting $x^{2}$ instead of $x$ in (3) we get

$$
D\left(x^{2 k}\right)=k x^{2 m} D\left(x^{2}\right) x^{2 n}
$$

for all $x \in R$. By (28) we infer

$$
\begin{aligned}
k x^{2 m}(n D(x) x+m x D(x)) x^{2 n} & =n D\left(x^{k}\right) x^{k}+m x^{k} D\left(x^{k}\right) \\
& =k n x^{m} D(x) x^{n+k}+k m x^{m+k} D(x) x^{n},
\end{aligned}
$$

which implies

$$
x^{2 m}(n D(x) x+m x D(x)) x^{2 n}=n x^{m} D(x) x^{n+k}+m x^{m+k} D(x) x^{n}
$$

for all $x \in R$. After the complete linearization of this identity and putting $x_{1}=x_{2}=x_{3}=x$ and $x_{4}=\ldots=x_{2 k}=c$ in the so obtained identity we get

$$
[[D(x), x], x]=0
$$

for all $x \in R$. By the result of Brešar ([6]) it follows

$$
[D(x), x]=0
$$

for all $x \in R$. Consequently, (28) can be written as

$$
D\left(x^{2}\right)=2 x D(x)=D(x) x+x D(x)
$$

for all $x \in R$. In other words, $D$ is a Jordan derivation. By Herstein's theorem $D$ is a derivation. Thus $D$ is a nonzero commuting derivation. By Posner's second theorem it follows that $R$ is commutative. Thereby the proof of the theorem is completed.

\section{REFERENCES}

[1] K. I. Beidar, M. Brešar and M. A. Chebotar, Functional identities revised: the fractional and the strong degree, Comm. Algebra 30 (2002), 935-969.

[2] K. I. Beidar and M. A. Chebotar, On functional identities and d-free subsets of rings I, Comm. Algebra 28 (2000), 3925-3951.

[3] K. I. Beidar and Y. Fong, On additive isomorphisms of prime rings preserving polynomials, J. Algebra 217 (1999), 650-667.

[4] K. I. Beidar, W. S. Martindale, III and A. V. Mikhalev, Rings with generalized identities, Marcel Dekker, Inc., New York, 1996.

[5] K. I. Beidar, A. V. Mikhalev and M. A. Chebotar, Functional identities in rings and their applications, Russian Math. Surveys 59 (2004), 403-428.

[6] M. Brešar, A generalization of the notion of centralizing mappings, Proc. Amer. Math. Soc. 114 (1992), 641-649.

[7] M. Brešar, Functional identities: A survey, Contemp. Math. 259 (2000), 93-109.

[8] M. Brešar and J. Vukman, On left derivations and related mappings, Proc. Amer. Math. Soc. 110 (1990), 7-16.

[9] Q. Deng, On Jordan left derivations, Math. J. Okayama Univ. 34 (1992), 145-147.

[10] E. C. Posner, Derivations in prime rings, Proc. Amer. Math. Soc. 8 (1957), 10931100 .

[11] L. H. Rowen, Some results on the center of a ring with polynomial identity, Bull. Amer. Math. Soc. 79 (1973), 219-223.

[12] J. Vukman, Commuting and centralizing mappings in prime rings Proc. Amer. Math. Soc. 109 (1990), 47-52. 
[13] J. Vukman, On left Jordan derivations of rings and Banach algebras, Aequationes Math. 75 (2008), 260-266.

[14] J. Vukman and M. Fošner, A characterization of two-sided centralizers on prime rings, Taiwanese J. Math. 11 (2007), 1431-1441.

[15] J. Vukman and I. Kosi-Ulbl, On some equations related to derivations in rings, Internat. J. Math. Math. Sci. 17 (2005), 2703-2710.

M. Fošner

Faculty of logistics

University of Maribor

Mariborska cesta 2, 3000 Celje

Slovenia

E-mail: maja.fosner@uni-mb.si

J. Vukman

Department of Mathematics and Computer Science

Faculty of natural sciences and mathematics

University of Maribor

Koroška cesta 160, SI-2000 Maribor

Slovenia

E-mail: joso.vukman@uni-mb.si

Received: 17.2.2010. 